\title{
How do parents and providers trade-off between disability and survival? Preferences in the treatment of pediatric medulloblastoma
}

This article was published in the following Dove Press journal:

Patient Preference and Adherence

\author{
Amir Khakban' \\ Tima Mohammadi \\ Larry D Lynd' \\ Don J Mabbott ${ }^{3,4}$ \\ Eric Bouffet ${ }^{5,6}$ \\ Louise Gastonguay' \\ Zafar Zafari ${ }^{7}$ \\ David Malkin ${ }^{5,6}$ \\ Michael D Taylor 8,9 \\ Carlo A Marra ${ }^{10}$
}

'Collaboration for Outcomes Research and Evaluation, Faculty of Pharmaceutical Sciences, The University of British

Columbia, Vancouver, BC, Canada;

${ }^{2}$ Centre for Health Evaluation and

Outcome Sciences, University of British

Columbia, St Paul's Hospital, Vancouver,

BC, Canada; ' ${ }^{3}$ Department of Psychology,

University of Toronto, Toronto, ON,

Canada; ${ }^{4}$ Department of Psychology,

The Hospital for Sick Children, Toronto,

ON, Canada; ${ }^{5}$ Department of Pediatrics,

University of Toronto, Toronto, ON,

Canada; ${ }^{6}$ Department of Haematology/

Oncology, The Hospital for Sick

Children, Toronto, ON, Canada; ${ }^{7}$ Health

Policy and Management, Mailman School

of Public Health, Columbia University,

New York, NY, USA; ${ }^{8}$ Departments

of Surgery, Laboratory Medicine and

Pathobiology, University of Toronto,

Toronto, ON, Canada; 'Department of

Neurosurgery, The Hospital for Sick

Children, Toronto, ON, Canada; ${ }^{10} \mathrm{~S} c h o o l$

of Pharmacy, University of Otago,

Dunedin, New Zealand

Correspondence: Carlo A Marra School of Pharmacy, University of Otago,

18 Frederick Street, Dunedin 901।,

New Zealand

Tel +64 34795044

Email carlo.marra@otago.ac.nz
Objective: The aim of this study was to determine the preferences of parents with children with medulloblastoma and clinicians who manage this condition and their trade-offs between survival and disability using a best-worst scaling (BWS) choice experiment.

Methods: Mixed methods were used to develop a BWS tool. Health professionals involved in the care of medulloblastoma patients were contacted through oncology networks in Canada. Parents of children diagnosed with brain tumors were recruited via two clinics in Vancouver and Toronto. The profile case BWS was used with each participant completing 12 choice profiles with the respondent indicating the best and worst features of each profile. Surveys were stratified into good, moderate, and poor prognosis based on the probability of survival presented. Paired model conditional logit analysis was used to generate quantitative preferences.

Results: Fifty-four parents ( $80 \%$ female) and 176 providers (36\% female, $79 \%$ oncologists) participated in this study. There were many similarities in the parents' and providers' preferences for treatment although the parents tended to value survival higher than disability while providers seemed to value the opposite. Specifically, providers were willing to take more risk of recurrence in a child with good prognosis compared to intermediate and poor prognosis. Also, parents were less willing to take more survival risks than providers when they had to trade-off between mild disability and survival rate.

Conclusion: This study provides useful insights into the preferences of parents and health care providers, the stakeholders of a collaborative decision for the treatment of pediatric medulloblastoma, and compares their values and trade-offs between different levels of survival and disability.

Keywords: best-worst scaling, BWS, preference, medulloblastoma, trade-off, cancer

\section{Introduction}

Medulloblastoma, the most frequent malignant brain tumor in children, has benefited from improvements in treatments over the past 20 years. With the current multimodal therapy which includes surgery, multi-agent chemotherapy, and radiation, the survival rate has improved significantly. ${ }^{1}$ However, the improvement in the survival rate has come with an increased incidence of neurodevelopmental adverse effects which gives rise to issues in cognitive and academic outcomes in children. ${ }^{2}$ Previous studies have shown that the severity of neurocognitive deficit is largely related to the dose of radiation that the child is exposed to as part of treatment. ${ }^{3,4}$ However, there has been a reluctance to reduce the radiotherapy dose due to the risks of treatment failure, higher recurrence, and lower survival rate. Recent studies have revealed that the genotype of a tumor can predict different prognoses for the disease which may form the basis for a reduction 
or elimination of radiation..$^{5-8}$ Several studies are ongoing to test the feasibility of a sub-group adapted strategy: SJMB12 has tailored postoperative treatment based on the subgrouping according to three different categories, namely WNT, Sonic Hedgehog (SHH) and non-WNT, non-SHH with specific doses of craniospinal radiation and specific chemotherapy regimen for each category. ${ }^{9}$ PNET5, the European protocol, classifies patients with average risk medulloblastoma into WNT and non-WNT groups. ${ }^{10}$ The Children's Oncology group has launched a protocol for patients with average risk WNT medulloblastoma, with the intent to decrease the dose of craniospinal to $18 \mathrm{~Gy} .{ }^{11}$ Additional subcategories may emerge, as the outcome of patients with high risk features, such as MYC amplification in group 3 or TP53 mutation in the $\mathrm{SHH}$ group is not satisfactory. ${ }^{12}$

However, because the predictive value of molecular testing is not always perfect, there are trade-offs between the accuracy of the test, survival, and disability in children with medulloblastoma. We have previously examined these trade-offs using qualitative research ${ }^{13}$ and using best-worst scaling (BWS) in a large sample of the general population. ${ }^{14}$ We recognize that preferences often differ between those who are directly affected by the disease (such as those with an affected child) and those who consider it hypothetically. As such, the objective of this paper was to determine the preferences of parents with children with medulloblastoma and clinicians who manage this condition and their trade-offs between survival and disability.

\section{Methods}

We used BWS, a choice-based method, to measure preferences based on random utility theory (RUT). ${ }^{15}$ The idea behind BWS is that people are able to choose the two items in a set of three or more choices that represent their extreme (lowest and highest) preferences. There are three types of BWS: object case, profile case, and multi-profile case BWS. ${ }^{16}$ We have used the profile case BWS in this study. In this framework, a specific set of attribute-levels (a profile) is presented to the respondents in each choice task, and they are asked to make choices within the profile to choose the best (or the most important) and the worst (or the least important) attribute level.

\section{Selection of attribute levels and scenarios}

We previously reported the preferences of members of the general public for the treatment of pediatric medulloblastoma. ${ }^{14}$ In brief, to identify the important aspects of the treatment for medulloblastoma, semi-structured focus groups were conducted with parents of children with medulloblastoma and health care providers. The results of the focus groups of 16 parents and 16 providers showed three important aspects: 1) the accuracy of the genetic test, 2) the adverse effects of the treatment on the child, and 3) the 10-year survival rate (Table 1). ${ }^{13}$ We stratified the BWS for three different scenarios based on the disease prognosis: good, intermediate, and poor prognosis with 10-year survival rates of $95 \%, 70 \%$, and $40 \%$, respectively.

To define the severity of the adverse treatment effects, we used Bloom's scale for levels of disability. ${ }^{17,18}$ To summarize, these levels of disability are as follows: 1) No disability: normal life defined by appropriate mental development and activities; 2) Mild disability: a) learning disabilities involving schooling with special services within the normal school system and clumsiness and b) mild difficulties in balance, running, and jumping; 3) Partial disability: a) capable of self-care if old enough with problems developing daily living skills like feeding oneself or getting dressed, and b) Overall evidence of intellectual impairment, severe learning

Table I Attributes and attribute levels

\begin{tabular}{llll}
\hline Attributes & Good prognosis & Intermediate prognosis & Poor prognosis \\
\hline Accuracy of test & $100 \%$ accuracy of test & $100 \%$ accuracy of test & $100 \%$ accuracy of test \\
& $95 \%$ accuracy of test & $95 \%$ accuracy of test & $95 \%$ accuracy of test \\
& $90 \%$ accuracy of test & $90 \%$ accuracy of test & $90 \%$ accuracy of test \\
Adverse effects of & $85 \%$ accuracy of test & $85 \%$ accuracy of test & $85 \%$ accuracy of test \\
treatment on the child & Child will have normal mental & Child will have normal mental & Child will have normal mental \\
& Chelopment & development & development \\
& Child will experience mild disability & Child will experience mild disability & Child will experience mild disability \\
& Child will experience severe disability & Child will experience severe disability & Child will experience severe disability \\
I0-year survival rate & $100 \%$ survival rate & $85 \%$ survival rate & $55 \%$ survival rate \\
& $95 \%$ survival rate & $70 \%$ survival rate & $40 \%$ survival rate \\
& $90 \%$ survival rate & $55 \%$ survival rate & $25 \%$ survival rate \\
& $80 \%$ survival rate & $40 \%$ survival rate & $10 \%$ survival rate \\
\hline
\end{tabular}


disabilities necessitating special schooling; or 4) Severe disability: a) incapable of self-care (not developing daily living skills like feeding oneself or getting dressed and child needs help for daily routines), b) necessitating schooling for the intellectually impaired; and c) inability to walk or eat without help.

\section{Experimental design}

The experimental design refers to the specific combinations of attributes and levels which respondents evaluate in their choice tasks with the overarching goal of estimating preferences from the least amount of choice sets answered. In order to elicit the most information possible and maximize the precision of estimated choice model parameters for a given number of choice questions, efficient experiment design methods have been used. ${ }^{19,20}$

As discussed in our previous article, ${ }^{14}$ in this study we used software from Sawtooth (Sawtooth Software, Inc. Sequim, WA, USA) to obtain optimal or near-optimal designs. ${ }^{21}$ This approach ensures that the experimental design is well balanced and orthogonal or near-orthogonal. It also considers connectivity and positional balance. In the final design, we controlled for the presence of the implausible combinations.

Before presenting the choice tasks, a brief background information and description of attribute levels in the BWS were provided to the respondents. Each questionnaire comprised 12 BWS choice tasks, and in each of them, respondents were inquired to identify their most and least preferable aspect of the treatment. An example of the choice tasks is presented in Figure 1.

\section{Sample}

Health professionals involved in the care of medulloblastoma patients were identified and contacted through pediatric oncology networks in Canada (ie, C17/CPHOD, Canadian Pediatric Brain Tumour Consortium) and IPSOS Reid Canada. These professionals received an email describing the purpose of the study along with a consent form and inviting them to complete the survey online. Parents of children diagnosed with brain tumors were recruited via British Columbia (BC) Children's Hospital in Vancouver and Sick Kids' Hospital in Toronto. Parents with children across all pediatric brain tumor types were included given the treatment and sequelae are similar across the various tumor types. We did not include parents of children who were currently undergoing treatment. They received an invitation letter containing the website address and a universal invitation code which was subsequently changed so that participants remained anonymous. After assessing their eligibility, parents were included in the study. All respondents provided written informed consent. This study was approved by the University of British Columbia Behavioural Ethics Committee (\#H1203528).

\section{Statistical analysis}

The BWS data were coded and analyzed using the paired model conditional logit analysis. In the paired model of the conditional logit framework, the observations are the unique pairs of the best-worst attribute levels.

In this framework, it is assumed that each respondent's utility of an alternative can be separated into a systematic part (observable and explainable) and a stochastic component which captures the unobservable or unexplainable

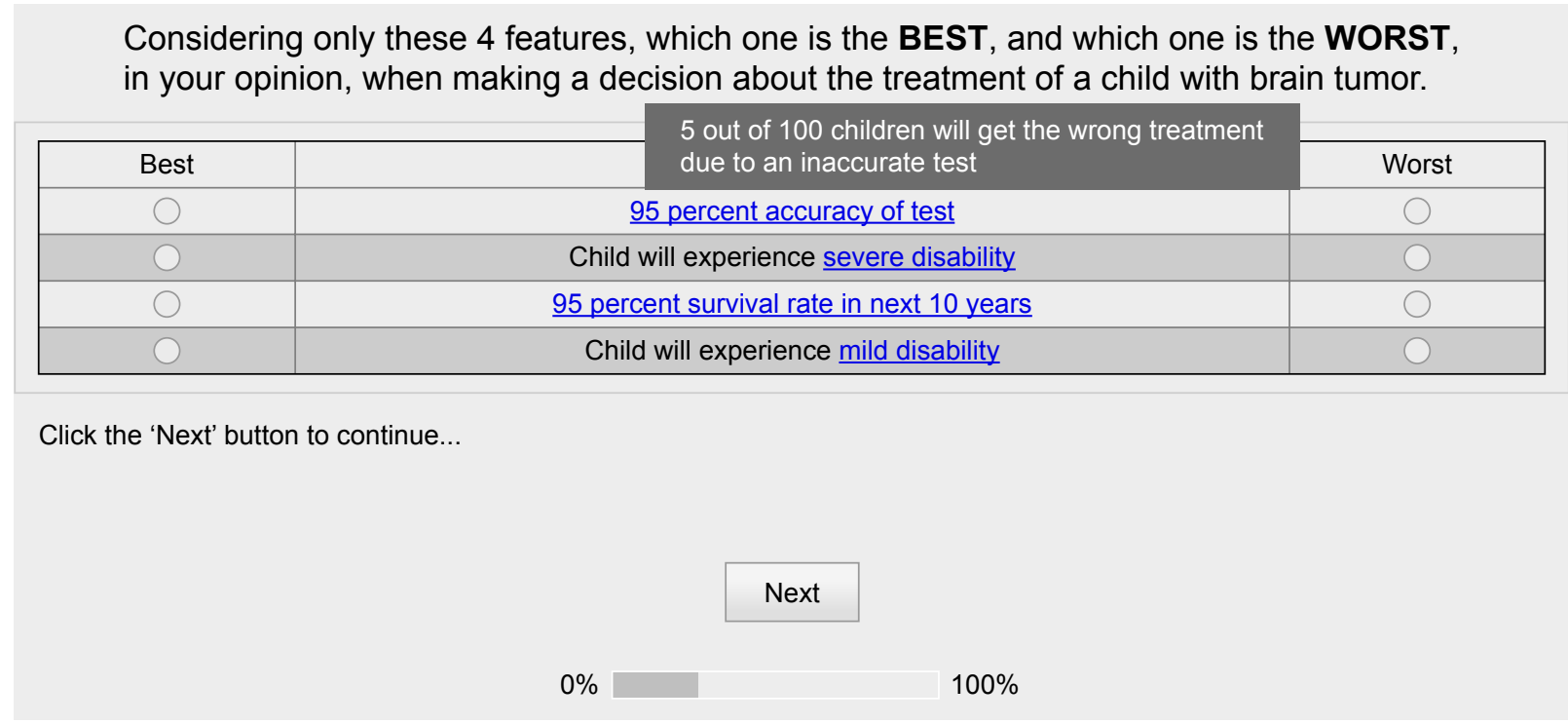

Figure I Choice task template. 
component of utility. The basic assumption of RUT is that the systematic component of utility can be considered as a function of the variables representing the attributes (features) of the alternative:

$$
U_{i j s}=\beta_{i}^{\prime} x_{i j s}+\varepsilon_{i j s}
$$

where $U_{i j s}$ is individual $i$ 's utility from alternative $j$ in choice set $s, \beta_{i}$ is a vector of individual $i$ 's utility parameter, $x_{i j s}$ is a vector of attributes associated with choice $j$, and $\varepsilon_{i j s}$ is a stochastic part of utility (error term). The assumption of an independently and identically extreme value error term can be used to develop the probability of choosing an alternative. In the paired conditional logit model, the units are pairs of attribute levels. From all possible pairs of attribute levels, individual $i$ chooses a pair which has the maximum differences in utility between its best and worst choices. ${ }^{22}$ The probability that respondent $i$ chooses attribute level $j$ as the best and attribute level $k$ as the worst one is:

$P($ pair $j$ and $k$ chosen as best - worst $)=\frac{e^{U_{i j}-U_{i k}}}{\sum_{m=1}^{M} \sum_{n=1}^{N} e^{U_{i m}-U_{i n}}}$

We also used "counts" or "frequencies" of BWS data to briefly explore the importance of attribute levels for the respondents. ${ }^{23}$ In this approach, count frequencies are used to calculate a best-minus-worst score for attribute levels in all choice sets. This score can be used as a descriptive statistic of the choice model. ${ }^{24}$

\section{Results}

Details of the demographics of the respondents are summarized in Table 2. A total of 54 parents (79\% female) and 176 providers (36\% female) responded to the questionnaire. The health care provider response rate to our questionnaire was $82 \%$ (through IPSOS Reid), whereas a response rate of $57 \%$ was achieved for parents of affected children.

Table 2 Demographic characteristics of the respondents

\begin{tabular}{lll}
\hline Variable & Parents & Providers \\
\hline Number of respondents & 54 & 176 \\
Female, N (\%) & $43(79.6)$ & $64(36.4)$ \\
Average age (SD) & $42.7(13.3)$ & $45.2(10.3)$ \\
Parents with university degree, N (\%) & $4 I(76)$ & - \\
Oncologists, N (\%) & - & $139(79)$ \\
Nurses and social workers, N (\%) & - & $10(6)$ \\
General physicians, N (\%) & - & $9(5)$ \\
Radiotherapists, N (\%) & - & $7(4)$ \\
Neurosurgeons, N (\%) & - & $3(1)$ \\
Unspecified, N (\%) & - & $8(5)$ \\
\hline
\end{tabular}

The distribution of brain tumors in the affected children of the included parents was medulloblastoma (44\%), glioma (15\%), ependymoma (7\%), and unspecified (34\%). In terms of treatment received, $68 \%$ of patients at least had chemotherapy, $75 \%$ had radiotherapy, and 78\% experienced surgery. About $51 \%$ of the patients received all three types of treatments. The mean age of the children during diagnosis was 6.36 years (SD, 3.73). The average age of children at the time of the BWS survey was 10.1 years (SD, 5.5).

The average age of parents and providers was 42.7 (SD, 13.3) and 45.2 (SD, 10.3) years, respectively. In each group of respondents (parents and providers), we estimated three separate models for each prognostic scenario and a model with pooled data of the two groups (parents and providers) with an interaction term of the dummy variable for the group and the attribute levels.

\section{Good prognosis}

Results showed that all the coefficients were statistically significantly different from zero for providers. For parents, except for the lowest level of the test accuracy and mild disability, all other attributes were significantly important (Table 3). The parents and providers followed the same pattern of preferences with an identical ranking for the first four, the most favorable attribute levels. However, the magnitudes of preferences were slightly different, and parents showed more variability across the levels. The attributes of "child with severe disability" and " $100 \%$ survival rate" were the least and the most favorable attribute levels in both the groups.

In terms of how the groups would trade-off between survival and disability, both parents and providers preferred an $80 \%$ survival rate over having a child with disability. Though, as it can be seen in Table 3, the parents' preferences for a "child with minor disability" was similar to " $80 \%$ survival rate."

We observed statistically significant differences in the magnitude of preferences between parents and providers for the first three levels of the test accuracy and the first two levels of the adverse effects of the treatments (Table 3 ). However, the relative importance of the accuracy of the test for providers was lower as compared to the parents.

\section{Intermediate prognosis}

As it is reported in Table 4, in the intermediate prognostic scenario, the attributes "child having normal mental development" following by " $100 \%$ accuracy of the test" had the most impact on parents' preferences. The attribute "child having normal mental development" was also the most favorable for health care providers followed by 

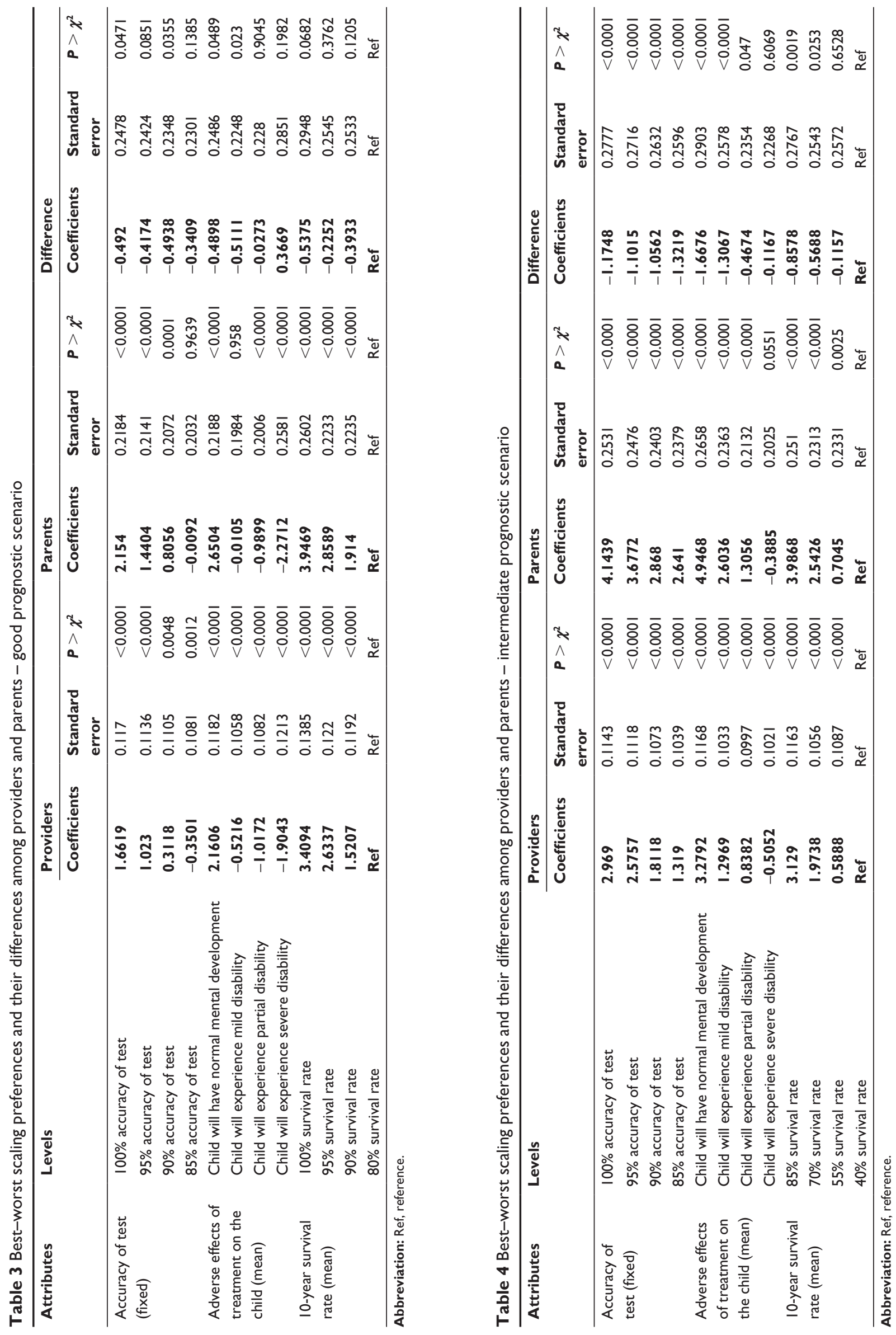
" $85 \%$ survival rate." The least favorable factor for both parents and providers was the attribute "child will experience severe disability." Table 4 highlights the comparison of providers' and parents' preferences toward attribute levels. As it can be seen from this table, the difference between coefficients representing preferences of parents and providers were not statistically significant for "child will experience severe disability" and " $55 \%$ survival rate." Though for others, we observed that providers have shown lower difference with the reference attribute level.

\section{Poor prognosis}

The results for the poor prognostic scenario are reported in Table 5. In this scenario, the attributes "child having normal mental development" and " $100 \%$ accuracy of the test" were the most favorable attribute levels for parents and providers. Providers ranked the attributes "child experiences severe disability" and " $10 \%$ survival rate" as the least favorable followed by " $25 \%$ survival rate." However, parents ranked the attributes " $10 \%$ survival rate," " $25 \%$ survival rate," and "child experience severe disability" as least favorable meaning that their preferences for having a child with severe disability were higher than for the $<25 \%$ survival rate. Providers, on the other hand, were willing to take the survival risk for avoiding severe disability. The estimated coefficient for the dummy variable representing that belonging to the parent or provider showed statistical significance only for attribute levels presenting the three levels of mental disability (mild, partial, and severe disability).

\section{Discussion}

To our knowledge, this study is the first to use a choice-based method to quantify and understand parents' and providers' preferences for the treatment of pediatric medulloblastoma and their willingness to trade survival and disability. This is an important issue, as medulloblastoma protocols are increasingly tailored according to molecular biology, and more specifically tumor subgrouping. While some protocols are attempting at improving the outcome of high-risk patients, others are planning to decrease the treatment intensity for lower risk patients, such as those diagnosed with WNT medulloblastoma. As a result some of these protocols may be associated with a higher risk of relapse.

The results showed that there were many similarities between the parents' and providers' preferences for treatment although the parents tended to value survival higher than disability, while providers seemed to value the opposite. Specifically, providers were willing to take more risk of recurrence in a child with good prognosis compared to intermediate

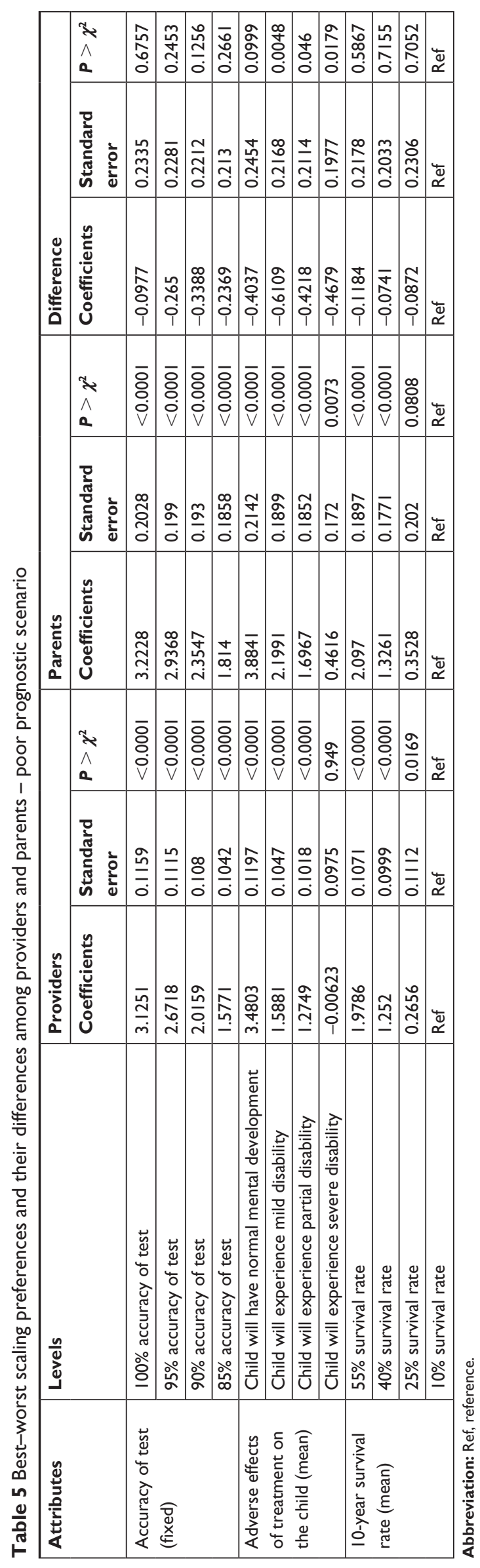


and poor prognosis. Also, parents were less willing to take more survival risks than providers when they had to trade-off between mild disability and survival rate.

Previous studies conducted to compare parents' and health care professional's preferences for pediatric cancer treatment have mostly used qualitative methods. ${ }^{25-27}$ Although these studies show similar findings to ours, there are advantages of using a choice-based methods such as respondents' preferences are quantitatively examined through multiple choice tasks and trade-offs among the attributes can be examined. As we used BWS which asks respondents to consider and choose between different key factors of the treatment (attribute level), the estimated preferences and the trade-offs between survival and disability may not be specific to pediatric medulloblastoma. These choices can be used to guide research in other pediatric areas where the process of decision-making for parents and clinicians also requires considering this trade-off. Also quantifying both parents' and health care providers' preferences using the same BWS questionnaire makes it possible to compare their perspectives toward different aspects of the treatments directly and with more accuracy.

In the literature, there have been very few studies that examine the trade-offs between health-related domains and survival that are made by patients and/or their families. A study by Slevin et $\mathrm{al}^{28}$ showed that most patients were willing to accept intensive chemotherapy with little chance of benefit in contrast to those without cancer (general public and health care providers). Similarly, a study by Bremnes et $\mathrm{al}^{29}$ showed that patients aged $<40$ years would accept toxic treatments for little chance of benefit including chance of cure ( $7 \%$ median), life prolongation ( 3 months), and symptom relief $(8 \%)$ and that health care providers (especially surgeons and surgical nurses) were unwilling to consider similar risks for little benefit. These findings are similar to that reported in pediatric cancers. Tomlinson et $\mathrm{al}^{26}$ showed that parents would consider chemotherapy even if quality of life was reduced (by a median of one hypothetical unit) and survival was decreased (by a median of 1 month), whereas the health care workers would require a median increase in survival by at least 2 months and/or an increase in quality of life by a median in two hypothetical units before initiating chemotherapy.

Daneault et $\mathrm{al}^{30}$ wrote about the role of "hope" in latestage cancers and how it influences the willingness to accept treatment (despite the expected lack of benefits) by patients. Generally, hope appears to be a dynamic construct and may be a way to help patients and their families to cope with a terminal diagnosis or a serious illness such as cancer. Hope was misguided most of the times. Doyle et $\mathrm{al}^{31}$ showed that patients believe that additional chemotherapy would be curative despite being informed that their condition was palliative and does not necessarily take the view of quality of life or societal costs into consideration. Thus, our research contributes to this knowledge base by specifically showing the quantitative trade-offs that parents are willing to take in even bleak scenarios.

There are some limitations in our study. First, the numbers of respondents in parents and providers groups were not equal and both sample sizes were limited. Second, like any other stated preferences method including probabilities (risk) as an attribute, respondents might have some difficulty understanding the choice task. ${ }^{32}$ Third, because of the limited number of potential respondents for this BWS, we cannot exclude selection bias in that those most motivated to respond may have different preferences than the population from which they were drawn. Finally, the over-representation of females in the parent group as compared to the provider group could be a potentially confounding factor if gender is associated with preferences. Unfortunately, we do not have sufficient male representation in the parent group to test this hypothesis and adjust accordingly.

\section{Conclusion}

In conclusion, the results of this study provide useful insights into the preferences of parents and health care providers, the stakeholders of a collaborative decision for pediatric medulloblastoma treatment, and compare their values and trade-offs between different levels of survival and disability. These findings can be used in conducting clinical investigations aimed at selecting options for children with different medulloblastoma prognosis.

\section{Acknowledgment}

This study was supported by an unrestricted grant from Genome Canada, Genome BC, and Genome Ontario.

\section{Disclosure}

The authors report no conflicts of interest in this work.

\section{References}

1. Turcotte LM, Neglia JP, Hayat MA. Survivors of childhood cancer: risk of new primary neoplasms of the CNS. In: Hayat MA, editor. Tumors of the Central Nervous System, Volume 12 [Internet]. Springer Netherlands; 2014 [cited 2017 Mar 10]:137-145. Available from: http://link.springer.com/ chapter/10.1007/978-94-007-7217-5_12. Accessed March 20, 2018.

2. Ris MD, Packer R, Goldwein J, Jones-Wallace D, Boyett JM. Intellectual outcome after reduced-dose radiation therapy plus adjuvant chemotherapy for medulloblastoma: a Children's Cancer Group study. J Clin Oncol. 2001;19(15):3470-3476. 
3. Grill J, Renaux VK, Bulteau C, et al. Long-term intellectual outcome in children with posterior fossa tumors according to radiation doses and volumes. Int J Radiat Oncol Biol Phys. 1999;45(1):137-145.

4. Kieffer-Renaux V, Bulteau C, Grill J, Kalifa C, Viguier D, Jambaque I. Patterns of neuropsychological deficits in children with medulloblastoma according to craniospatial irradiation doses. Dev Med Child Neurol. 2000;42(11):741-745.

5. Northcott PA, Shih DJ, Peacock J, et al. Subgroup-specific structural variation across 1,000 medulloblastoma genomes. Nature. 2012; 488(7409):49-56.

6. Cho YJ, Tsherniak A, Tamayo P, et al. Integrative genomic analysis of medulloblastoma identifies a molecular subgroup that drives poor clinical outcome. J Clin Oncol. 2011;29(11):1424-1430.

7. Kool M, Koster J, Bunt J, et al. Integrated genomics identifies five medulloblastoma subtypes with distinct genetic profiles, pathway signatures and clinicopathological features. PLoS One. 2008;3(8):e3088.

8. Ellison D. Classifying the medulloblastoma: insights from morphology and molecular genetics. Neuropathol Appl Neurobiol. 2002; 28(4):257-282.

9. A clinical and molecular risk-directed therapy for newly diagnosed medulloblastoma. Available from: https://clinicaltrials.gov/ct2/show/ NCT01878617. NLM identifier: NCT01878617. Accessed May 28, 2018.

10. International society of paediatric oncology (SIOP) PNET 5 medulloblastoma. Available from: https:/clinicaltrials.gov/ct2/show/ NCT02066220. NLM identifier: NCT02066220. Accessed May 28, 2018.

11. Reduced craniospinal radiation therapy and chemotherapy in treating younger patients with newly diagnosed WNT-Driven medulloblastoma. Available from: https:/clinicaltrials.gov/ct2/show/NCT02724579. NLM identifier: NCT02724579. Accessed May 28, 2018.

12. Ramaswamy V, Remke M, Bouffet E, et al. Risk stratification of childhood medulloblastoma in the molecular era: the current consensus. Acta Neuropathol. 2016;131(6):821-831.

13. Henrich N, Marra CA, Gastonguay L, et al. De-escalation of therapy for pediatric medulloblastoma: trade-offs between quality of life and survival. Pediatr Blood Cancer. 2014;61(7):1300-1304.

14. Khakban A, Mohammadi T, Lynd LD, et al. Societal preferences in the treatment of pediatric medulloblastoma: balancing risk of death and quality of life. Pediatr Blood Cancer. 2017;64(6):e26340.

15. Finn A, Louviere JJ. Determining the appropriate response to evidence of public concern: the case of food safety. J Public Policy Mark. 1992; 11(2):12-25.

16. Flynn TN, Louviere JJ, Peters TJ, Coast J. Estimating preferences for a dermatology consultation using best-worst scaling: comparison of various methods of analysis. BMC Med Res Methodol. 2008;8(1):76.

17. Bloom HJ, Wallace EN, Henk JM. The treatment and prognosis of medulloblastoma in children. a study of 82 verified cases. Am J Roentgenol Radium Ther Nucl Med. 1969;105(1):43-62.
18. Bloom HJG, Glees J, Bell J. The treatment and long-term prognosis of children with intracranial tumors: a study of 610 cases, 1950-1981. Int J Radiat Oncol Biol Phys. 1990;18(4):723-745.

19. Mühlbacher AC, Kaczynski A, Zweifel P, Johnson FR. Experimental measurement of preferences in health and healthcare using best-worst scaling: an overview. Health Econ Rev. 2016;6(1):2.

20. Cheung KL, Wijnen BF, Hollin IL, et al. Using best-worst scaling to investigate preferences in health care. Pharmacoeconomics. 2016; 34(12):1195-1209.

21. Proceedings of the Sawtooth Software Conference. 2000 Proceedings. pdf [Internet] [cited 2017 Mar 24]. Available from: http:/www.sawtoothsoftware.com/download/techpap/2000Proceedings.pdf\#page=175. Accessed March 20, 2018.

22. Marley AAJ, Louviere JJ. Some probabilistic models of best, worst, and best-worst choices. J Math Psychol. 2005;49(6):464-480.

23. Marley AAJ, Pihlens D. Models of best-worst choice and ranking among multiattribute options (profiles). J Math Psychol. 2012;56(1):24-34.

24. Hess S, Daly A. Handbook of Choice Modelling. Northampton, MA: Edward Elgar Publishing; 2014:721.

25. Mack JW, Cook EF, Wolfe J, Grier HE, Cleary PD, Weeks JC. Understanding of prognosis among parents of children with cancer: parental optimism and the parent-physician interaction. J Clin Oncol. 2007; 25(11):1357-1362.

26. Tomlinson D, Bartels U, Hendershot E, Maloney A-M, Ethier M-C, Sung L. Factors affecting treatment choices in paediatric palliative care: Comparing parents and health professionals. Eur J Cancer. 2011; 47(14):2182-2187.

27. Tomlinson D, Bartels U, Gammon J, et al. Chemotherapy versus supportive care alone in pediatric palliative care for cancer: comparing the preferences of parents and health care professionals. CMAJ. 2011; 183(17):E1252-E1258.

28. Slevin ML, Stubbs L, Plant HJ, et al. Attitudes to chemotherapy: comparing views of patients with cancer with those of doctors, nurses, and general public. BMJ. 1990;300(6737):1458-1460.

29. Bremnes RM, Andersen K, Wist EA. Cancer patients, doctors and nurses vary in their willingness to undertake cancer chemotherapy. Eur J Cancer Oxf Engl 1990. 1995;31A(12):1955-1959.

30. Daneault S, Dion D, Sicotte C, et al. Hope and noncurative chemotherapies: which affects the other? J Clin Oncol. 2010;28(13):2310-2313.

31. Doyle C, Crump M, Pintilie M, Oza AM. Does palliative chemotherapy palliate? Evaluation of expectations, outcomes, and costs in women receiving chemotherapy for advanced ovarian cancer. J Clin Oncol. 2001; 19(5):1266-1274

32. Miron-Shatz T, Hanoch Y, Graef D, Sagi M. Presentation format affects comprehension and risk assessment: the case of prenatal screening. J Health Commun. 2009;14(5):439-450.
Patient Preference and Adherence

\section{Publish your work in this journal}

Patient Preference and Adherence is an international, peer-reviewed, open access journal that focuses on the growing importance of patient preference and adherence throughout the therapeutic continuum. Patient satisfaction, acceptability, quality of life, compliance, persistence and their role in developing new therapeutic modalities and compounds to optimize
Dovepress

clinical outcomes for existing disease states are major areas of interest for the journal. This journal has been accepted for indexing on PubMed Central. The manuscript management system is completely online and includes a very quick and fair peer-review system, which is all easy to use. Visit http://www dovepress.com/testimonials.php to read real quotes from published authors. 